\title{
Effect of abdominal binder after laparoscopic cholecystectomy on enhanced recovery: a randomized controlled trial
}

\author{
Hyung Uk Choi, Sang Hun Park, Eo Jin San Lee, Kyung Uk Jung, Jun Ho Shin, Sung Ryol Lee \\ Department of Surgery, Kangbuk Samsung Hospital, Sungkyunkwan University School of Medicine, Seoul, Korea
}

Purpose: The purpose of this randomized controlled trial was to compare the effects of abdominal binder after laparoscopic cholecystectomy.

Methods: From August to December 2020, 66 patients who were set to undergo cholecystectomy were selected for a prospective trial at Kangbuk Samsung Hospital, Seoul, Republic of Korea, and their clinical characteristics and postoperative surgical outcomes were evaluated. Among 66 patients, 33 patients belong to the abdominal binder group and the other 33 patients belong to the control group.

Results: The average hospital stay was $2.46 \pm 1.29$ days, and was not significantly different between the two groups. The average postoperative pain score (visual analogue scale, $0-10$ ) 12, 24, and 48 hours after surgery were not significantly different. However, the degree of comfort score was significantly higher for the control group patients $(2.56$ vs. $3.33, p<0.001)$. Time to the first ambulation, walking ability, return of bowel function, time to full diet resumption, and the numbers of analgesics and antiemetics administered were not significantly different between the two groups.

Conclusion: No postoperative recovery benefit and no reduction in hospital stay was found in patients who used an abdominal binder while undergoing laparoscopic cholecystectomy. Statistically, between the group that used the binder and the one that did not, no significant differences in surgical outcome nor postoperative outcome were observed. The only exception was that the degree of comfort score was significantly higher in the control group. Therefore, in terms of patient benefit and convenience, wearing an abdominal binder after laparoscopic cholecystectomy is not recommended.
Received March 23, 2021

Revised 1st April 1,2021 2nd May 17, 2021 3rd May 24, 2021 4th May 27, 2021

Accepted May 28, 2021

Corresponding author Sung Ryol Lee

Department of Surgery, Kangbuk Samsung Hospital, Sungkyunkwan University School of Medicine, 29 Saemunan-ro, Jongno-gu, Seoul 03181, Korea

Tel: $+82-2-2001-8404$

Fax: +82-2-2001-2131

E-mail: sungryol.lee@samsung.com ORCID:

https://orcid.org/0000-0001-9963-3673

Keywords: Laparoscopic cholecystectomy, General surgery, Laparoscopy, Pain

This is an Open Access article distributed under the terms of the Creative Commons Attribution Non-Commercial License (http:// creativecommons.org/licenses/by-nc/4.0/) which permits unrestricted non-commercial use, distribution, and reproduction in any medium, provided the original work is properly cited.
Copyright $\odot$ The Korean Society of Endoscopic and Laparoscopic Surgeons.

\section{INTRODUCTION}

Patients who undergo abdominal surgery can incur postoperative complications such as abdominal pain, nausea, vomiting, wound infection, pulmonary complications, or incisional hernia [1-3]. Postoperative complications can increase hospitalization length, reduce patient quality of life, and even cause permanent defects. Therefore, an abdominal binder is commonly and routinely used after abdominal surgery to prevent postoperative complications [4-7]. An abdominal binder covers the whole abdomen and it helps relieve pain, provides support for deep breathing exercise, and promotes walking ability [8,9]. Several studies have found that the use of an abdominal binder after abdominal surgery, including cesarean section and cardiac surgery, may be efficacious for reducing postoperative complications [4-7]. However, Huang et al. [10] are reluctant to have their patients use 
the abdominal binder because it increases abdominal pressure and, thus, increases the risk of poor pulmonary function [11] and deep vein thrombosis $[10,12]$. Skeptical surgeons also argue that the abdominal binder has no effect on reducing postoperative complications [13-15]. Moreover, the use of an abdominal binder after laparoscopic surgery may be unnecessary and uncomfortable for patients and can interrupt diet resumption. There have been reports that abdominal binder use after laparoscopic or open surgery both positively [4-9] and negatively [10-12] affects postoperative respiratory function, walking performance, and pain but no study has evaluated binder use solely for laparoscopic cholecystectomy surgery. Therefore, we performed a randomized controlled trial to evaluate the effect of abdominal binders after laparoscopic cholecystectomy by comparing postoperative outcomes in two patient groups especially to identify the difference of visual analogue scale (VAS) score (0-10) between the abdominal binder group and control group after laparoscopic cholecystectomy.

\section{MATERIALS AND METHODS}

\section{Patients}

This is a randomized controlled trial to investigate if abdominal binder use after laparoscopic cholecystectomy has an impact on postoperative recovery.
From August 2020 to December 2020, 66 patients who were set to receive a cholecystectomy were selected for a prospective trial at Kangbuk Samsung Hospital in Seoul, Republic of Korea. Patients scheduled for laparoscopic cholecystectomy between August and December 2020 were screened for the following criteria: 18 to 65 years old and American Society of Anesthesiologists (ASA) physical status (PS) classification I and II. The exclusion criteria were a history of ventral hernia, walking disability, chronic obstructive respiratory disease, malignancy, chronic pain syndrome, open conversion, or inability to complete the questionnaire. A total of 265 patients underwent cholecystectomy surgeries during that period, and 62 were screened out due to old age ( $>65$ years), 127 patients refused to be included in the study, and 10 patients were excluded because of their previous histories (ventral hernia, 3; chronic obstructive pulmonary disease, 4; or malignancy, 3). Finally, 65 patients were selected. The selected patients were randomly assigned to the abdominal binder group or the control group in a 1:1 ratio (Fig. 1). A permuted block randomization algorithm was used via an interactive web-based response system (http://randomization.com). Recorded patient characteristics were age, sex, history of previous abdominal surgery, body mass index (BMI), and comorbidities (Table 1), while the surgical outcome parameters were operation time, open conversion rate, postoperative hospital stay, postoperative complications, and mortality (Table 2).

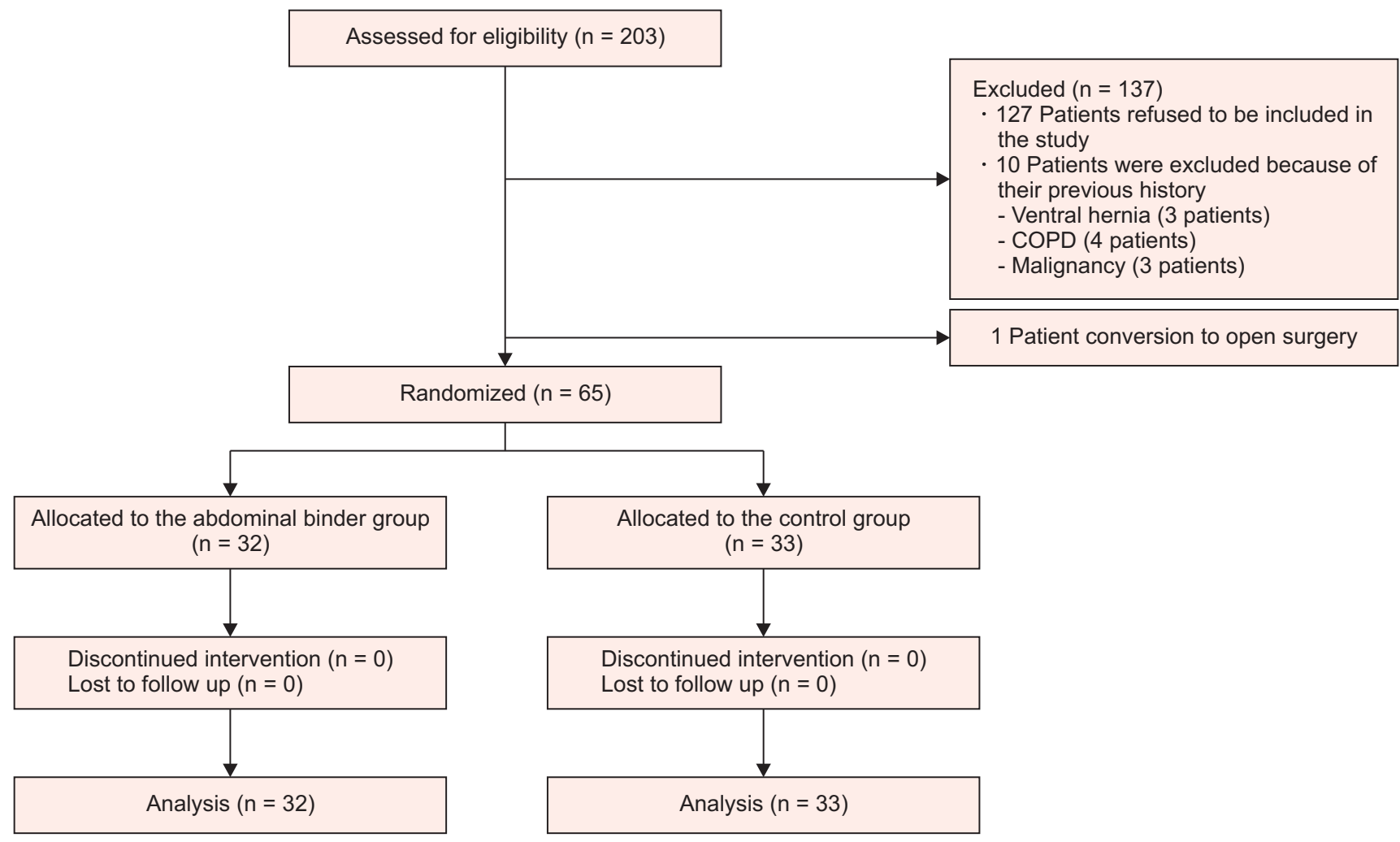

Fig. 1. Details of flow charts for study patients. COPD, chronic obstructive pulmonary disease. 
Table 1. Patient characteristics

\begin{tabular}{lcccc}
\hline \multicolumn{1}{c}{ Variable } & Total & Binder & Control & $p$ value \\
\hline No. of patients & 65 & 32 & 33 & \\
\hline Age (yr) & $47.05 \pm 10.15$ & $46.97 \pm 10.43$ & $47.12 \pm 10.04$ & 0.952 \\
\hline Sex & & & & 0.460 \\
Female & $33(50.7)$ & $18(56.2)$ & $15(45.5)$ & \\
Male & $32(49.2)$ & $14(43.8)$ & $18(54.5)$ & \\
Underlying disease & & & & 0.114 \\
Diabetes mellitus & $4(6.2)$ & $0(0)$ & $4(12.1)$ & 0.258 \\
Hypertension & $8(12.3)$ & $2(6.3)$ & $6(18.2)$ & 0.492 \\
Hepatitis B virus & $1(1.5)$ & $1(3.1)$ & $0(0)$ & $>0.999$ \\
Pulmonary disease & $3(4.6)$ & $1(3.1)$ & $2(6.1)$ & $>0.999$ \\
Angina & $0(0)$ & $0(0)$ & $0(0)$ & $>0.999$ \\
Cerebrovascular accident & $0(0)$ & $0(0)$ & $0(0)$ & 0.708 \\
Others & $7(10.8)$ & $4(12.5)$ & $3(9.0)$ & 0.107 \\
Previous operation & $13(20.0)$ & $9(28.1)$ & $4(12.1)$ & 0.404 \\
Body mass index $\left(k g / m^{2}\right)$ & $24.66 \pm 3.63$ & $25.05 \pm 4.13$ & $24.29 \pm 3.09$ & 0.722 \\
ASA PS classification & & & & \\
I & $48(73.8)$ & $23(71.9)$ & $25(75.8)$ & $8(24.2)$ \\
II & $17(26.2)$ & $9(28.1)$ & & 0.623 \\
Pathology & & & $4(12.1)$ & \\
GB polyp & $8(12.3)$ & $4(12.5)$ & $2(6.0)$ & \\
GB adenomyosis & $2(3.1)$ & $0(0)$ & $0(0)$ & \\
Chronic cholecystitis & $54(83.1)$ & $1(3.1)$ & \\
Acute cholecystitis & $1(1.5)$ & & & \\
\hline
\end{tabular}

Data are expressed as mean \pm standard deviation or number (\%).

ASA, American Society of Anesthesiologists; PS, physical status; GB, gallbladder.

\section{Treatment}

This study was designed as a single-center, prospective, randomized, parallel-group, phase IV, single-blind trial involving one pancreaticobiliary surgeon, with more than 5,000 laparoscopic cholecystectomies.

A single surgeon was selected to minimize variation in surgery performance. The cholecystectomy was performed using the three-port method, with the patient placed on the operating table in a supine position. A pneumoperitoneum was made through an anterior approach at the umbilicus with an 11-mm trocar. Next, two 5-mm trocars were inserted into the right subcostal region at the midclavicular line and at the subxiphoid area. The gallbladder was pulled out through the umbilical incision line and the umbilical fascia was closed with a 2-0 vicryl suture. Subcostal and xiphoid trocar sites were closed by subcuticular suture with a 3-0 vicryl. An abdominal drain (Jackson-Pratt drain) was inserted in all patients. In the abdominal binder group, all patients used a 22-cm height binder (Se-jung Inc., Seoul, Korea) (Fig. 2). The binder was placed across the incision line and the patients were instructed to use the binder for at least two consecutive days after surgery. Patients in both groups received the same postoperative management. The patients with the postoperative fluid collection were treated by percutaneous drainage. Patients were discharged after successful ambulation, return of bowel function, and full diet resumption. All patients were scheduled for follow-up 1 week after the discharge.

\section{Statistical analyses}

The sample size was determined from 20 patients who underwent laparoscopic surgery in Kangbuk Samsung Hospital, and 
Table 2. Surgical outcomes

\begin{tabular}{|c|c|c|c|c|}
\hline Variable & Total $(n=65)$ & Binder $(n=32)$ & Control $(n=33)$ & $p$ value \\
\hline Operation time (min) & $40.54 \pm 8.93$ & $41.41 \pm 10.26$ & $39.70 \pm 7.49$ & 0.445 \\
\hline Blood loss (mL) & $37.85 \pm 11.92$ & $40.31 \pm 10.62$ & $35.45 \pm 12.77$ & 0.101 \\
\hline Changed hemoglobin & $0.58 \pm 0.50$ & $0.64 \pm 0.58$ & $0.53 \pm 0.41$ & 0.366 \\
\hline Transfusion & $0(0)$ & $0(0)$ & $0(0)$ & $>0.999$ \\
\hline Open conversion or additional trocar & $0(0)$ & $0(0)$ & $0(0)$ & $>0.999$ \\
\hline Postoperative complication & & & & 0.492 \\
\hline No complication & $64(98.5)$ & $31(96.9)$ & $33(100)$ & \\
\hline Bleeding & $0(0)$ & $0(0)$ & $0(0)$ & \\
\hline Bile leakage & $0(0)$ & $0(0)$ & $0(0)$ & \\
\hline Fluid collection & $1(1.5)$ & $1(3.1)$ & $0(0)$ & \\
\hline Pneumonia & $0(0)$ & $0(0)$ & $0(0)$ & \\
\hline Pleural effusion & $0(0)$ & $0(0)$ & $0(0)$ & \\
\hline Angina & $0(0)$ & $0(0)$ & $0(0)$ & \\
\hline Wound infection & $0(0)$ & $0(0)$ & $0(0)$ & \\
\hline Hernia & $0(0)$ & $0(0)$ & $0(0)$ & \\
\hline Other & $0(0)$ & $0(0)$ & $0(0)$ & \\
\hline
\end{tabular}

Data are expressed as mean \pm standard deviation or number (\%).

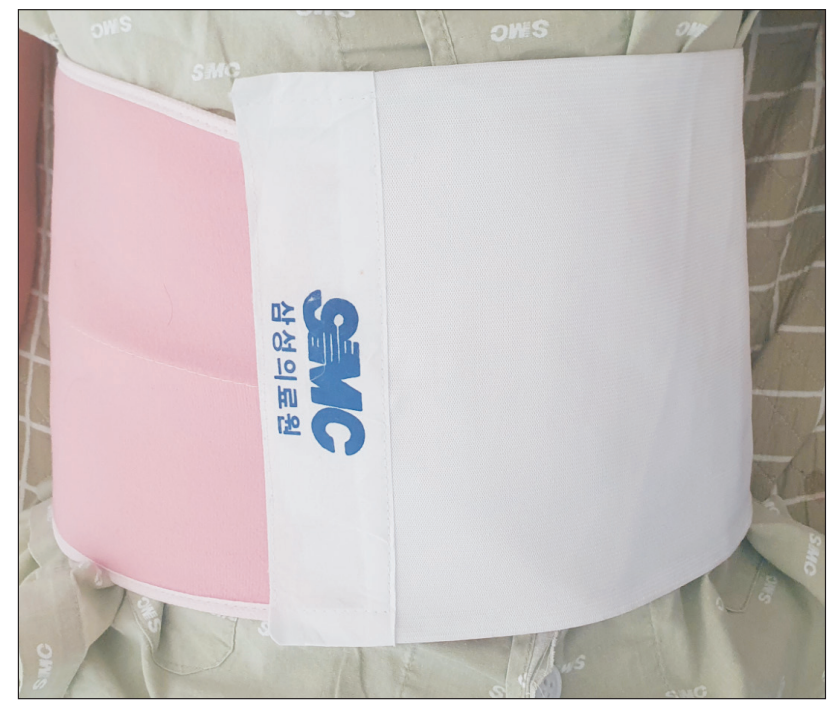

Fig. 2. Picture of abdominal binder used for patients.

they were checked for their pain score (VAS score) 24 hours after surgery. The patients who did not use an abdominal binder after surgery and the data were gathered retrospectively (mean \pm standard deviation $[\mathrm{SD}]$ of VAS, $3.2 \pm 1.4$ ). For power calculation, equal standard deviation was assumed for the binder group. We also assumed that the VAS score was better in the binder group and a 1-point difference in VAS score was clinically meaningful.
Considering a 5\% significance level and an $80 \%$ power level, each group required at least 31 patients. And considering 5\% elimination, our final number of research participants (66 patients, 33 patients/group; $\fallingdotseq 32.387$ ) was adequate.

For continuous variables, data are presented as mean \pm SD and for categorical variables, data are presented as frequency (percentage). For comparisons between the two groups using the Student $t$ test. Categorical variables were subjected to the chisquare analysis or Fisher exact test, as appropriate. A two-sided $p$ value of $<0.05$ was deemed a showing statistical significance. All the analyses were performed with IBM SPSS version 24.0 (IBM Corp., Armonk, NY, USA).

\section{Outcome measures}

Patients were requested to complete a questionnaire prior to discharge about their pain, degree of comfort, time to ambulation by own power, walking ability, return of bowel function, and lapse of time before full diet resumption. Patients were asked to rate their pain score using the VAS 12, 24, and 48 hours after surgery $[8,9]$. Degree of comfort and walking ability were subjectively reported by the patients. Questionnaire response options ranged from very comfortable (5) to very uncomfortable (0). Assuming that the patient's normal walking performance was 10 , they were asked how it was 48 hours after surgery. To evaluate bowel function, bowel sounds and time to flatulence were moni- 
tored. The numbers of analgesics (tramadol $50 \mathrm{mg}$ intravenously, pro re nata) and antiemetics (metoclopramide $10 \mathrm{mg}$ intravenously, pro re nata) taken were recorded from medical charts.

\section{RESULTS}

Table 1 shows patient characteristics for both the binder group and the control group. There are no meaningful baseline differences between the two groups. The average ages of the binder group and the control group were 46.97 and 47.12 years, respectively ( $p=0.952$ ). Differences in sex, comorbidities, history of previous abdominal surgery, BMI, and ASA PS classifications were not statistically significant. Surgical procedures were the same for all patients. Surgical outcomes are shown in Table 2, and operation time, blood loss, hemoglobin changes after surgery, transfusion, additional trocar, postoperative complications, and pathologies were not different between the two groups. Open conversion occurred in one patient in the binder group. It was impossible to dissect Calot's triangle because of severe adhesion, so that patient was excluded.

A postoperative complication occurred in one patient in the binder group who had postoperative fluid collection $(p=0.492)$.
The patient was discharged nine days after surgery. There were no mortality events in either group. Table 3 shows the differences in the postoperative outcomes for the two groups. The average hospital stays were 2.56 days in the binder group and 2.36 days in the control group, which were not significantly different. The average postoperative pain score (VAS) 12 hours after surgery was $6.41 \pm 1.21$ in the binder group and $6.27 \pm 1.13$ in the control group $(p=0.647)$. There were also no significant pain score differences 24 and 48 hours after surgery $(p=0.177$ and $p=0.58$, respectively). However, degree of comfort score was higher for the control group patients ( 2.56 vs. 3.33, $p<0.001$; Table 3, Fig. 3 ). Time to the first ambulation, walking ability, return of bowel function, time to full diet resumption, and the numbers of analgesics and anti-emetics used were not significantly different between the two groups.

\section{DISCUSSION}

For decades, surgeons have used abdominal binders to help prevent postoperative complications and relieve pain [4-7]. However recently, binder use has become controversial because of the lack of evidence for its effectiveness and due to its disadvantages

Table 3. Postoperative outcomes

\begin{tabular}{|c|c|c|c|c|}
\hline Variable & Total $(n=65)$ & Binder $(n=32)$ & Control $(n=33)$ & $p$ value \\
\hline Postoperative hospital stay (day) & $2.46 \pm 1.29$ & $2.56 \pm 1.41$ & $2.36 \pm 1.17$ & 0.538 \\
\hline \multicolumn{5}{|l|}{ VAS score } \\
\hline After $12 \mathrm{hr}$ & $6.34 \pm 1.16$ & $6.41 \pm 1.21$ & $6.27 \pm 1.13$ & 0.647 \\
\hline After $24 \mathrm{hr}$ & $3.46 \pm 1.32$ & $3.69 \pm 1.45$ & $3.24 \pm 1.17$ & 0.177 \\
\hline After $48 \mathrm{hr}$ & $1.11 \pm 1.13$ & $1.19 \pm 1.28$ & $1.03 \pm 0.98$ & 0.580 \\
\hline Degree of comfort & $2.95 \pm 0.86$ & $2.56 \pm 0.72$ & $3.33 \pm 0.82$ & $<0.001$ \\
\hline Degree of comfort & & & & 0.006 \\
\hline 0 & $0(0)$ & $0(0)$ & $0(0)$ & \\
\hline 1 & $2(3.0)$ & $2(6.2)$ & $0(0)$ & \\
\hline 2 & $16(24.6)$ & $12(37.5)$ & $4(12.1)$ & \\
\hline 3 & $33(50.7)$ & $16(50.0)$ & $17(51.5)$ & \\
\hline 4 & $11(16.9)$ & $2(6.2)$ & $9(27.2)$ & \\
\hline 5 & $3(4.6)$ & $0(0)$ & $3(9.0)$ & \\
\hline No. of analgesics used & $2.46 \pm 1.44$ & $2.22 \pm 1.43$ & $2.7 \pm 1.42$ & 0.182 \\
\hline No. of antiemetics used & $0.18 \pm 0.43$ & $0.25 \pm 0.51$ & $0.12 \pm 0.33$ & 0.233 \\
\hline Time to ambulation (hr) & $20.34 \pm 3.80$ & $20.19 \pm 4.67$ & $20.48 \pm 2.79$ & 0.757 \\
\hline Walking ability $(0-10)$ & $6.45 \pm 0.97$ & $6.28 \pm 0.89$ & $6.61 \pm 1.03$ & 0.179 \\
\hline Time to diet resumption (hr) & $28.95 \pm 5.87$ & $28.38 \pm 6.11$ & $29.52 \pm 5.68$ & 0.438 \\
\hline Time to first flatus (hr) & $21.20 \pm 3.84$ & $20.5 \pm 4.06$ & $21.88 \pm 3.53$ & 0.149 \\
\hline
\end{tabular}

Data are expressed as mean \pm standard deviation or number (\%).

VAS, visual analogue scale. 


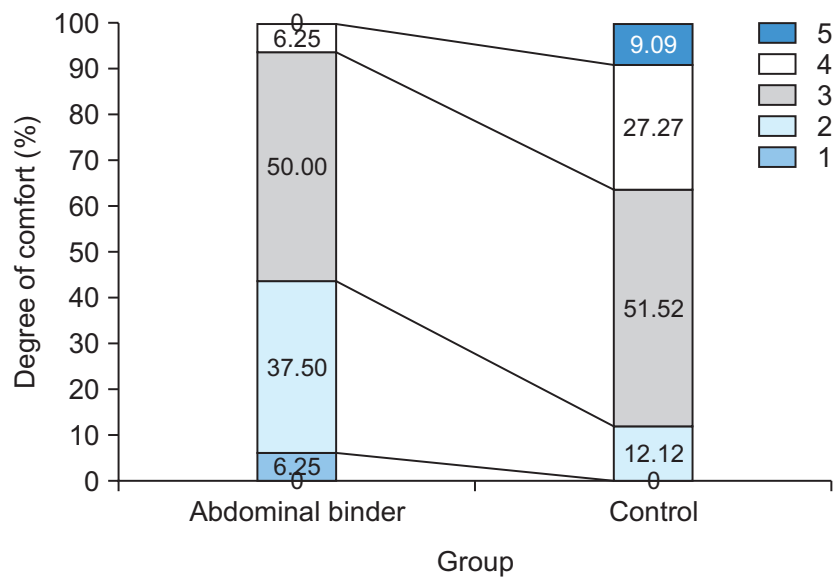

Fig. 3. Comparison of degree of comfort between the abdominal binder group and the control group.

[13-15]. The purpose of this randomized controlled trial was to compare the effects of an abdominal binder after laparoscopic cholecystectomy on pain, degree of comfort, time until ambulation, walking ability, return of bowel function, and diet resumption. The study's findings, consistent with previous studies, reveal that there are no statistically significant differences between the binder group and the control group. In our study, degree of comfort was more improved for patients in the control group (2.56 vs. 3.33, $p<0.001$ ). This finding was consistent with previous studies. In a trial, Ali et al. [16] reported that an abdominal binder for open cholecystectomy showed no differences in reducing pain than without the binder. The disadvantage of wearing an abdominal binder is that consistently compressing the abdomen can have a negative impact by increasing intraabdominal pressure. Some studies have reported that the increased intraabdominal pressure can reduce respiratory function [11] and can also cause deep vein thrombosis $[10,12]$ by decreasing venous return [17]. Surgical outcomes such as operation time, blood loss, hemoglobin changes after surgery, transfusion, additional trocar, postoperative complications, and pathologies were not significantly different between the two groups. These results show that routine use of abdominal binder after surgery is unnecessary. In terms of patient benefit and convenience, wearing an abdominal binder after laparoscopic cholecystectomy is not recommended.

The limitation of this study is that there are various cholecystectomy methods, like single port, three-port, or four-port laparoscopic or robotic surgeries, and we cannot apply our results to all laparoscopic cholecystectomies. The surgeries were performed by one surgeon in the same setting, so our results can be generalized to at least three-port cholecystectomies. In future studies, we hope to include these data and also evaluate other types of surgeries, such as laparoscopic appendectomy and herniorrhaphy, or different cholecystectomy approaches (single-port or four-port).
In conclusion, neither any postoperative recovery benefit nor reduced hospital stays were found in the patients who, after undergoing laparoscopic cholecystectomy, used an abdominal binder. On the other hand, degree of comfort score was significantly higher for the control group. Therefore, in terms of patient benefit and convenience, wearing an abdominal binder after laparoscopic cholecystectomy is not recommended. Additional studies encompassing larger patient groups with other types of laparoscopic surgeries and more institutions are required.

\section{NOTES}

\section{Ethical statements}

The study was conducted in compliance with the principles of the Declaration of Helsinki. The study protocol was reviewed and approved by our Institutional Review Board (No. 2020-02041-002). Detailed informed consent was obtained from all study participants. All clinical, operative, and follow-up data were documented in a structured master datasheet. The NCT number is NCT 04787458 . This manuscript was written according to CONSORT guidelines.

\section{Authors' contributions}

Conceptualization: SRL

Data curation: HUC, SHP

Investigation: HUC, EJSL

Methodology: SRL, JHS

Project administration: KUJ

Formal analysis, Visualization: All authors

Writing-original draft: All authors

Writing-review \& editing: All authors

All authors read and approved the final manuscript.

\section{Conflict of interest}

All authors have no conflicts of interest to declare.

\section{ORCID}

Hyung Uk Choi, https://orcid.org/0000-0002-3833-4597

Sang Hun Park, https://orcid.org/0000-0002-7519-6015

Eo Jin San Lee, https://orcid.org/0000-0002-3046-096X

Kyung Uk Jung, https://orcid.org/0000-0002-8844-3724

Jun Ho Shin, https://orcid.org/0000-0002-0712-2794

Sung Ryol Lee, https://orcid.org/0000-0001-9963-3673 


\section{REFERENCES}

1. Toner A, Hamilton M. The long-term effects of postoperative complications. Curr Opin Crit Care 2013;19:364-368.

2. Rezaiguia S, Jayr C. Prevention of respiratory complications after abdominal surgery. Ann Fr Anesth Reanim 1996;15:623-646.

3. Shea RA, Brooks JA, Dayhoff NE, Keck J. Pain intensity and postoperative pulmonary complications among the elderly after abdominal surgery. Heart Lung 2002;31:440-449.

4. Cheifetz O, Lucy SD, Overend TJ, Crowe J. The effect of abdominal support on functional outcomes in patients following major abdominal surgery: a randomized controlled trial. Physiother Can 2010;62:242-253.

5. Bouvier A, Rat P, Drissi-Chbihi F, et al. Abdominal binders after laparotomy: review of the literature and French survey of policies. Hernia 2014;18:501-506.

6. Saeed S, Rage KA, Memon AS, et al. Use of abdominal binders after a major abdominal surgery: a randomized controlled trial. Cureus 2019;11:e5832.

7. Szender JB, Hall KL, Kost ER. A randomized-clinical trial examining a neoprene abdominal binder in gynecologic surgery patients. Clin Exp Obstet Gynecol 2014;41:525-529.

8. Kliethermes C, Blazek K, Ali K, Nijjar JB, Kliethermes S, Guan X. A randomized controlled trial for abdominal binder use after laparoendoscopic single-site surgery. J Minim Invasive Gynecol 2018;25:842847.

9. Ghana S, Hakimi S, Mirghafourvand M, Abbasalizadeh F, Behnam- pour N. Randomized controlled trial of abdominal binders for postoperative pain, distress, and blood loss after cesarean delivery. Int J Gynaecol Obstet 2017;137:271-276.

10. Huang GJ, Bajaj AK, Gupta S, Petersen F, Miles DA. Increased intraabdominal pressure in abdominoplasty: delineation of risk factors. Plast Reconstr Surg 2007;119:1319-1325.

11. Obeid F, Saba A, Fath J, et al. Increases in intra-abdominal pressure affect pulmonary compliance. Arch Surg 1995;130:544-548.

12. Matarasso A. Venous thrombosis and tight underwear. Arch Intern Med 1996;156:214

13. Fagevik Olsén M, Josefson K, Wiklund M. Evaluation of abdominal binder after major upper gastrointestinal surgery. Adv Physiother 2009;11:104-10.

14. Christoffersen MW, Olsen BH, Rosenberg J, Bisgaard T. Randomized clinical trial on the postoperative use of an abdominal binder after laparoscopic umbilical and epigastric hernia repair. Hernia 2015;19:147-153.

15. Rothman JP, Gunnarsson U, Bisgaard T. Abdominal binders may reduce pain and improve physical function after major abdominal surgery: a systematic review. Dan Med J 2014;61:A4941.

16. Ali J, Serrette $C$, Khan T. The effect of abdominal binders on postoperative pulmonary function. Infect Surg 1983;2:875-881.

17. Willenberg T, Clemens R, Haegeli LM, Amann-Vesti B, Baumgartner I, Husmann M. The influence of abdominal pressure on lower extremity venous pressure and hemodynamics: a human in-vivo model simulating the effect of abdominal obesity. Eur J Vasc Endovasc Surg 2011;41:849-855. 